\title{
DHM and serious games: a case-study oil and gas laboratories
}

\author{
Santos, $\mathrm{V}^{\mathrm{a},{ }^{*}}$, Zamberlan, $\mathrm{M}^{\mathrm{b}}$, Streit, $\mathrm{P}^{\mathrm{b}}$, Oliveira, $\mathrm{J}^{\mathrm{b}}$, Guimarães, $\mathrm{C}^{\mathrm{b}}$, Pastura, $\mathrm{F}^{\mathrm{b}}$ and Cid, $\mathrm{G}^{\mathrm{b}}$ \\ ${ }^{a}$ Pontificia Universidade Católica do Rio de Janeiro - PUC-Rio. Rua Marquês de São Vicente, 225, Gávea, Rio de \\ Janeiro, RJ - Brasil - 22451-900 \\ ${ }^{\mathrm{b}}$ Instituto Nacional de Tecnologia, INT. Avenida Venezuela, 82, Rio de Janeiro, RJ. Brasil - 20081-312
}

\begin{abstract}
The aim in this paper is to present a research on the application of serious games for the design of laboratories in the oil and gas industries. The focus is in human virtual representation acquired from 3D scanning, human interaction, workspace layout and equipment designed considering ergonomics standards. The laboratory studies were simulated in Unity3D platform, which allows the users to control the $\mathrm{DHM}^{1}$ on the dynamic virtual scenario, in order to simulate work activities. This methodology can change the design process by improving the level of interaction between final users, managers and human factor teams. That helps to better visualize future work settings and improve the level of participation between all stakeholders.
\end{abstract}

Keywords: digital human model, simulation, virtual environment

\section{Introduction}

The research in serious games has been a focus issue for Government and corporate organizations, applied on training, simulation and education. There is a clear need for considering new frameworks, theories, methods and design strategies for making serious game applications and virtual world technologies more effective and useful as part of education, health and training. Virtual simulation has been used in Ergonomics for the design of control centers, transport design and product evaluation. (SANTOS, V., et al. 2009, SANTOS,V. et al. 2008, GUIMARÃES, C. P. et al. 2010).

This paper presents a project in which virtual reality and game engines are used to improve the workflow and interaction between teams in the design process of a series of oil and gas laboratories. This interactive environments provides the possibility of realistic scenario based drills among the use of DHM built from 3D anthropometric data and MOCAP, aiming to evaluate layout proposals, training new personnel based on the simulation of activities performed by the workers themselves .

The project is based in the research center of one of the biggest oil company in the world. In the stages of the project were considered: (1) gathering of laboratories and personnel data among its diagnosis based on ergonomic analysis, which provides the multidisciplinary team with data and knowledge on the procedures, in order to build new work environment layouts, (2) 3D scanning of the workers in several postures aiming the design of workstations, proposals of new working conditions and the development of their DHM for the virtual interactive environment and (3) MOCAP of the same workers performing daily activities in order to have their DHM with life like movements in the environment.

\section{Materials and methods}

\footnotetext{
*Venétia Santos. E-mail: ergon@ergonprojetos.com.br.
} 
The development of the simulators were segmented in a couple of stages: (1) building the 3D environment based on 2D CAD representation of the new proposal, (2) creation a furniture and workstations database with ergonomic recommendations and the application of both, Brazilian and International standards, (3) creation of an equipment database, which are based on real equipments located in the laboratories - dimensions, utilities, textures, etc., (4) development of the DHM with 3D scanning and MOCAP technologies and (5) implementation in the game engine with scripts and other setups.

Each 2D CAD proposal is transformed into 3D models (Figure 1), where later in Unity3D are applied interactive elements such as doors, drawers and windows, external scenarios, materials and textures, character controllers' built in options, and others, so that the environment is as realistic as possible. (Figure 2).

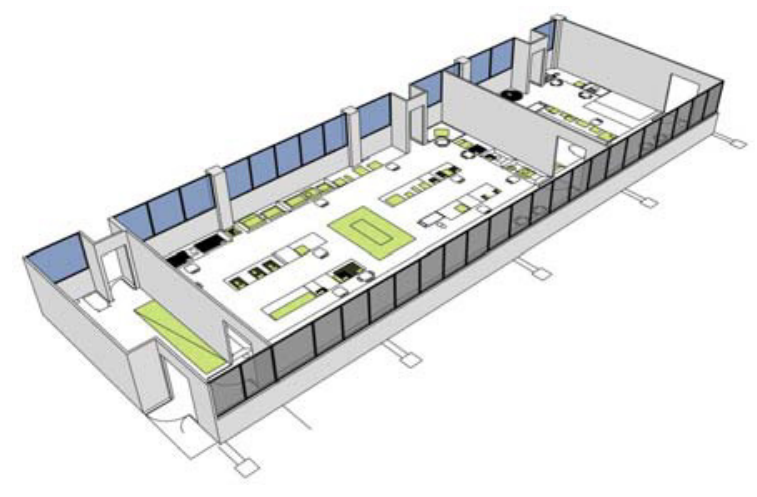

Fig. 1. 3D model based on 2D CAD floorplan

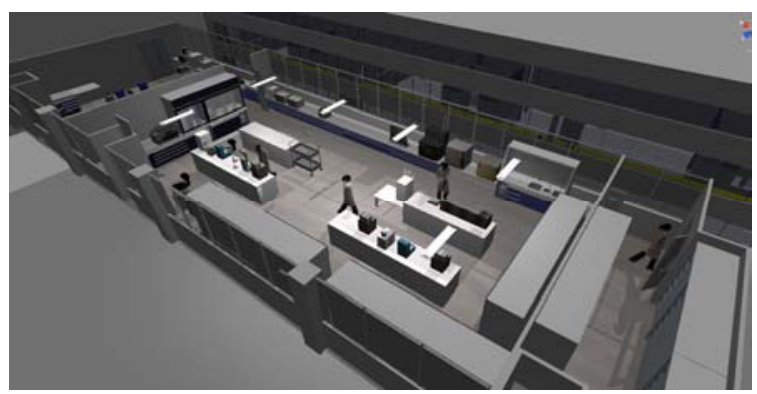

Fig. 2. 3D environment in Unity3D

Furniture and workstations present in the virtual laboratory are reproduced based on ergonomics recommendations and standards, modeled in
SketchUp software and implemented in a database among 3D equipment and other instruments (Figure 3). The $3 \mathrm{D}$ database allows direct exporting to the game platform in *.FBX format. The software also allows the segmentation of interactive components in groups, which in Unity3D can be easily set up by its hierarchy characteristic. It also exports their textures in *.JPEG, which are reproduced using their own pictures as textures (Figure 4), providing clearer understanding and faster recognition of each equipment by their respective users.

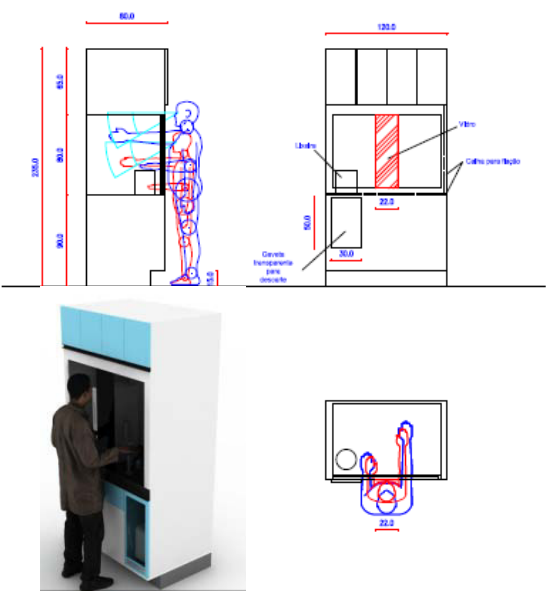

Fig. 3. Furniture recommendations

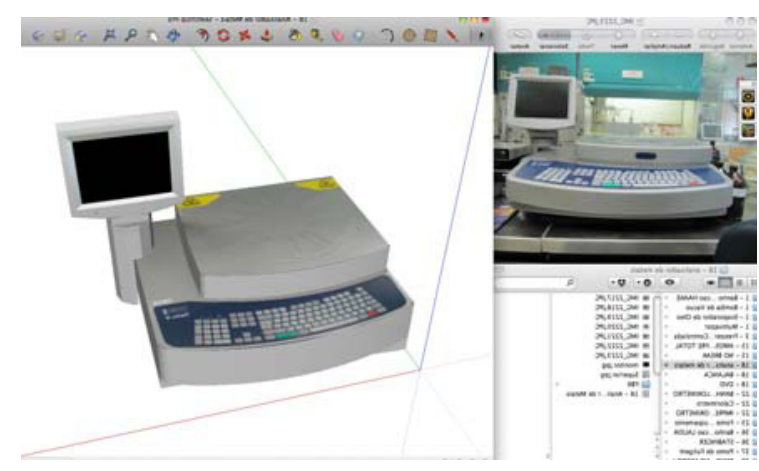

Fig. 4. Laboratory's equipment 3D database.

DHM used in the simulation have been obtained starting from high resolution DHM (more than one million vertex) obtained by $3 \mathrm{D}$ scanning of employees using "Cyberware Whole Body Color 3D Scanner" and "Cyberware Head and Face Color 3D Scanner" (Figure 5). 
To import the avatars into the virtual work environment in the Unity3D, their meshes must be seriously reduced. The high poly scan is imported into a $3 \mathrm{D}$ software to make a manual re-topology -
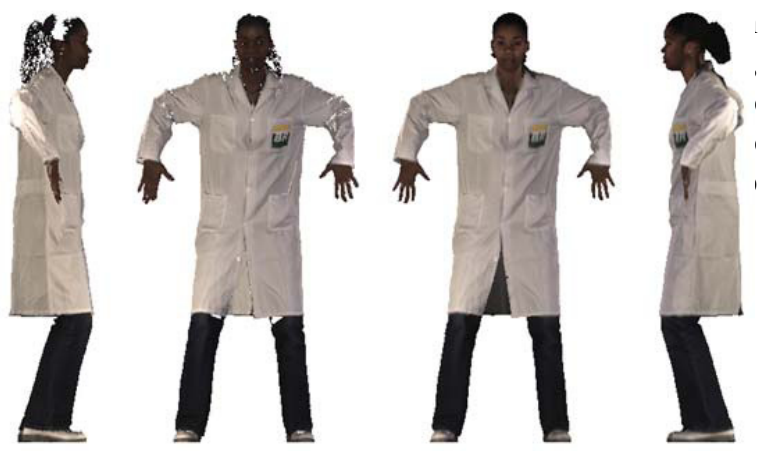

Fig. 5. Mesh hole filling in the high poly model and final low poly model

The result of such a process is a realistic virtual representation of the user (low poly and detailed mesh of the user) that respects with high fidelity the morphology of the person that was scanned.

To generate a planar texture which can be edited in graphic software, the process of retopology must consider the following step of marking the loops for UV mapping, so it can be opened properly (Figure 6).

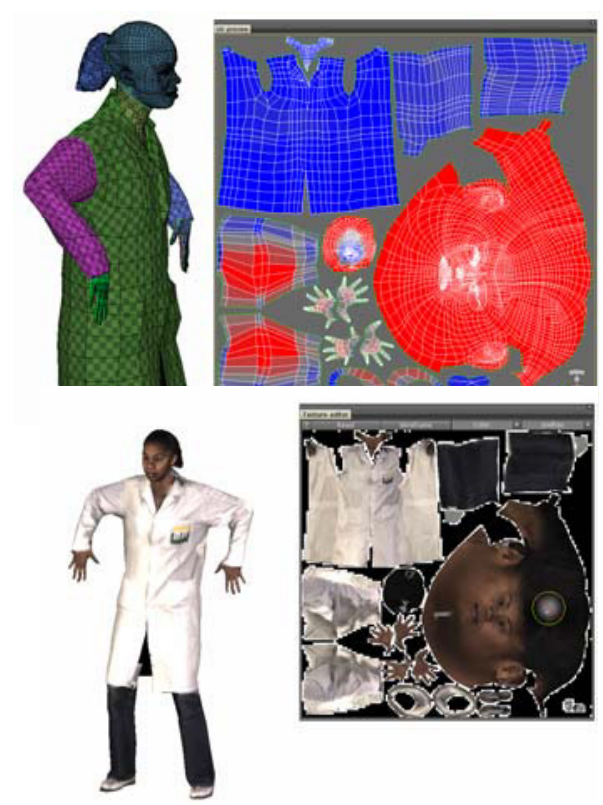

Fig. 6. Low poly model UV mapping and painting
To apply the texture in the low poly model, there are two approaches: (1) consists in the capturing photographs during the scanning process in order to have several pictures in different angles, which provides better resolution and (2) rendering the high poly model, which enables sectioning the model in order to have hidden surface images, such as armpits, inner legs, top and bottom views (Figure 7).

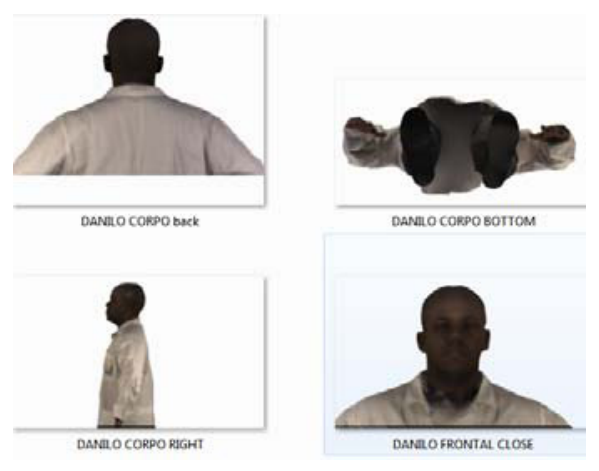

Fig. 7. DHM rendering to acquire texture images.

Most of the animations were acquired using "Moven" motion capture suite. The MOCAP sessions took place in the laboratory with personnel performing daily activities. The mocap files were exported to an animation format recognized by 3D Max (*.bvh format), then were edited and imported into the digital human models (Figure 8). In this process, the low poly model (*.obj format) is imported into 3D Max, where the biped structure is placed, correctly skinned to respond to each bone movement and exported to MotionBuilder.

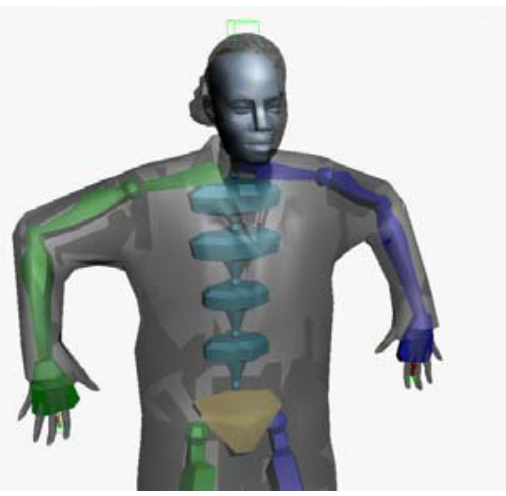

Fig. 8. Biped structure placed in the low poly model to be skinned. 
Inside MotionBuilder, the biped structure is characterized to follow the bones naming conventions and the motion capture files are imported, animations are then divided into full animation and cycle animations, as walk and idle, animations that play on loop, avoiding that the animation loading takes too much time.

After all animations are correctly set to its character, the animation is plotted to the skeleton and the DHM is sent back to 3D Max were the empty skeleton animation will be updated for those sent by MotionBuilder. At this time the file is already set to be exported to Unity with its correct media and animation.

\section{Results}

One of the simulator's goal is to allow multiple users to interact with each other using their own avatars. It will provide the team the ability to train activities, learn more about how their colleagues proceed and train new personnel.

In the final steps of the development of the project, the network function is set among all the $3 \mathrm{D}$ models. This setup involves: (1) scripting the objects (most of them are in java script language - e.g. interactive doors, drawers, equipments, picking up and dropping objects), (2) scripting the scene - menus, cameras, interactive cameras, networking, illumination, layout, possibility to enable or disable a trail renderer in order to map users paths across the laboratory. (Figure 9).

All these components are brought to the users through a friendly GUI (Graphic User Interface), which makes the simulator as intuitive as possible, so the users can focus on the activities and not on how to work with the engine.

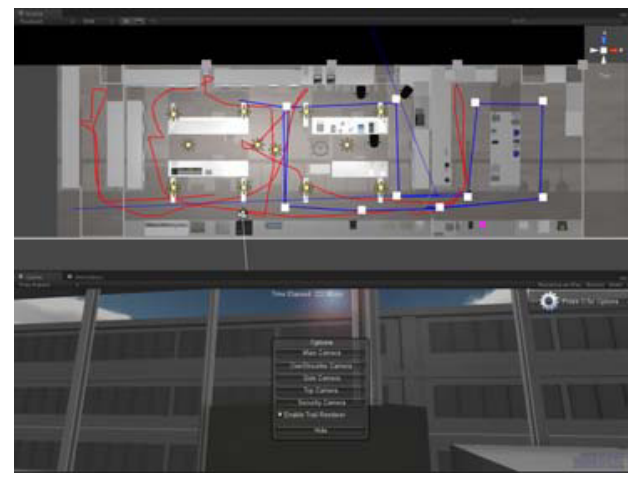

Fig. 9. Simulator's GUI.

\section{Conclusion}

Nowadays, industrial projects are developed with the use of 3D software engines instead of 2D tools, allowing the main focus to be human labor and not only the project itself. Allying it to the study of social interactions at work helps to project better environments.

The use of virtual environments gives the possibility to discuss, change, create and deliver a better result as it is more graphic and visual for non architects and designers professionals, to understand and discuss the new layout of the work space where can be chosen a better design alternative, optimize the interfaces, integrate countless projects and a great number of professionals involved.

Transparency of the future project allows adjustments and error recovery throughout the design process. Projects become more robust, since the scenarios and future activities may be simulated and also the risks involved studied.

These simulations may be used to evaluate technology, industrial safety and/or human performance. One may map process risks, ergonomic and architectural problems, escape routes, displacement of people in crisis situations, assembly and maintenance problems.

Therefore the conclusion is that virtual simulators of social interactions contribute towards: the activity of designers in the occupation of three-dimensional space; evaluation of possible alternatives; detailing the environment; validation of the future project by users, managers, and others; safety, health and environment evaluation; training of human resources.

As observed in this work, this simulation platform can be used by engineers in the design of future laboratories, thus minimizing the time required for similar projects and increasing the compliance of these environments to standards.

Even if character interaction cannot always be done in the virtual space, stakeholders can easily project their knowledge of the working situation in order to assess part of the new working space characteristics improving the participative dimension of the project.

Organizational decisions were taken around these tools; they help people to project themselves in their future working spaces and furthermore it was a great tool to improve the feeling of participation. 


\section{References}

[1] V. Santos; M.C.P.L. Zamberlan; P.; B. Pavard; P. Streit; J.L. Oliveira; C.P. Guimarães; F.C.H. Pastura, Social Interaction Simulators: Serious games for the design of complex sociotechnical systems. DHM 2011 - First International Symposium on Digital Human Modeling, UCBL - Université Claude-Bernard Lyon, 2011

[2] V. Santos; M.C.P.L. Zamberlan; P.; B. Pavard, Confiabilidade Humana e Projeto Ergonômico de Centros de Controle de Processos de Alto Risco, ed., Synergia, Rio de Janeiro, 2009.
[3] V. Santos; C. Guimarães; G. Cid, Simulação Virtual e Ergonomia, XV Congresso Brasileiro de Ergonomia, VI Fórum Brasileiro de Ergonomia, Porto Seguro, 2008.

[4] C. Guimarães; F.C.H. Pastura; B. Pavard; N. Pallamin; G. Cid; V. Santos; M.C.P.L. Zamberlan, Ergonomics Design Tools Based on Human Work Activities, 3D Human Models and Social Interaction Simulation IHX Congress, Miami, 2010. 